\title{
Cirrhosis Liver Classification on B-Mode Ultrasound Images by Convolution Neural Networks with Augmented Images
}

\author{
Yoshihiro Mitani, Robert B. Fisher, Yusuke Fujita, Yoshihiko Hamamoto, and Isao Sakaida
}

\begin{abstract}
In the medical imaging field, it is desirable to develop computer-aided diagnosis (CAD) systems. They are useful as a second opinion, and to objectively and quantitatively make diagnoses. In this study, we focus on liver ultrasound images. The cirrhosis liver is expected to progress to a liver cancer in the worst case. Therefore, we are investigating a CAD system to identify the cirrhosis liver sooner. In this paper, in order to classify cirrhosis or normal liver on regions of interest (ROIs) image from B-mode ultrasound images, we have proposed to use a convolution neural network $(\mathrm{CNN})$. CNNs are one of promising techniques for medical image recognition. In a previous study, we tried to classify the cirrhosis liver using a Gabor features based method, a higher order local auto-correlation (HLAC) feature based approach and an improved version. However, the classification performance of our preliminary experimental results were poor. The average error rates were still over $40 \%$. In order to more accurately classify the cirrhosis liver, we have explored the use of the CNNs. The experimental results show the effectiveness of the CNNs. Furthermore, by a data augmentation technique, the classification performance of the CNNs is improved.
\end{abstract}

Index Terms-Augumented images, perspective transformation, B mode ultrasound images, cirrhosis liver classification, computer-aided diagnosis system, convolution neural networks, over-training problem.

\section{INTRODUCTION}

In the medical imaging field, it is desirable to develop computer-aided diagnosis (CAD) [1] systems which can give a second opinion objectively and quantitatively. In this study, we focus on liver ultrasound images. Ultrasound images are widely used for diagnosis of liver cirrhosis [2]. The cirrhosis liver is expected to progress to liver cancer in the worst case. Therefore, we are investigating a CAD system to diagnose a cirrhosis liver sooner [3]-[6]. Fig. 1 shows B-mode ultrasound images. In the center, we can see the whole of the liver areas. Fig. 1(a) is a normal liver, (b) is a cirrhosis liver. Cirrhosis is a kind of disease, abnormal situation. The cirrhosis liver becomes harder compared with the normal one In this study we focus on regions of interest (ROIs) instead of the whole ultrasound image. We firstly consider examining small sized ROI images. Secondly from the experimental results of the ROI images, we try examining the whole sized ultrasound image. Fig. 2 shows ROI images. The ROI images

Manuscript received June 14, 2019; revised May 13, 2020

Yoshihiro Mitani is with the National Institute of Technology, Ube College, Japan (e-mail: mitani@ube-k.ac.jp)

Robert B. Fisher is with the University of Edinburgh, UK.

Yusuke Fujita, Yoshihiko Hamamoto, and Isao Sakaida are with the Yamaguchi University, Japan. are manually cut out from within the liver areas, by the physician. Fig. 2(a) shows examples of normal liver. On the other hand, (b) shows cirrhosis examples. The size of the ROI images is the same, $32 \times 32$ pixels. This is a typical 2-class problem, normal or abnormal. In this paper, we have focused the classification of these ROI images.

In detection of ROIs, Y. Fujita et al proposed a method of cirrhosis detection by using multiple ROIs voting with AdaBoost [3]. We also proposed a method based on the multiple ROIs combination by product rule [4], which achieved a better classification performance than multiple ROIs voting [3]. The blood vessel exclusion and rejection option were introduced for classification to select ROIs automatically [4]. The method with automatic ROI definition performed as good as manual ROI definition. We further proposed a training sample selection by self-training for multiple ROI classification to detect cirrhosis liver [5]. We see that a manually ROI definition and sample selection are important to improve classification accuracy in cirrhosis detection using ultrasound images. We also see that the quality of training samples is more important than the size of training samples from the experimental results.

In a previous study [6], we explored cirrhosis liver classification using a Gabor features based method and a higher order local autocorrelation (HLAC) feature based approach. These features are expected to fit the texture recognition problem. Our ultrasound ROI images are considered to be a kind of texture. We want to investigate which feature is the best for classifying ROI images, because the better features are, the better the classification performance is. In a conventional approach, such as the Gabor and HLAC features approach, it is important to find better features. However, the experimental results of this conventional approach were poor, with even the best performance. The average error rate was still over $40 \%$. Unfortunately we cannot easily classify the normal or cirrhosis. This might be a difficult pattern recognition problem.

In this paper, we have investigated the use of convolution neural networks (CNNs). CNNs are considered to be one of promising techniques in the image recognition field. CNNs originate from the artificial neural networks [7], and are highly suitable for images. The performance of CNNs has been improved by adding more layers and by deeply training to networks [8], [9]. CNNs are successfully applied in the pattern recognition field, especially in the image recognition field. Recently, CNNs are reported to be used widely in the medical imaging field [10]. With CNNs, we don't need to consider what features or what classifier we should use. 
Though we are usually concerned about these kinds of choices, CNNs can automatically connect between input and output. Although now we have the new issue of choosing the network model, network configuration, hyperparameters and training process. We are hoping that the deep nets will be able to improve performance in this difficult cirrhosis pattern recognition problem. However, CNNs need many training samples. There is also an over-training problem. This means that classification on the training samples is almost perfect, but test sample performance is low. From our preliminary experiments, we hypothesize that the $\mathrm{CNN}$ can memorize the class for every sample, as for ROIs images. The number of available samples is limited. It's known as the small sample size problem in the pattern recognition field [11], [12]. Therefore, we also investigate using an augmentation of the ROI images such as the perspective transformation.

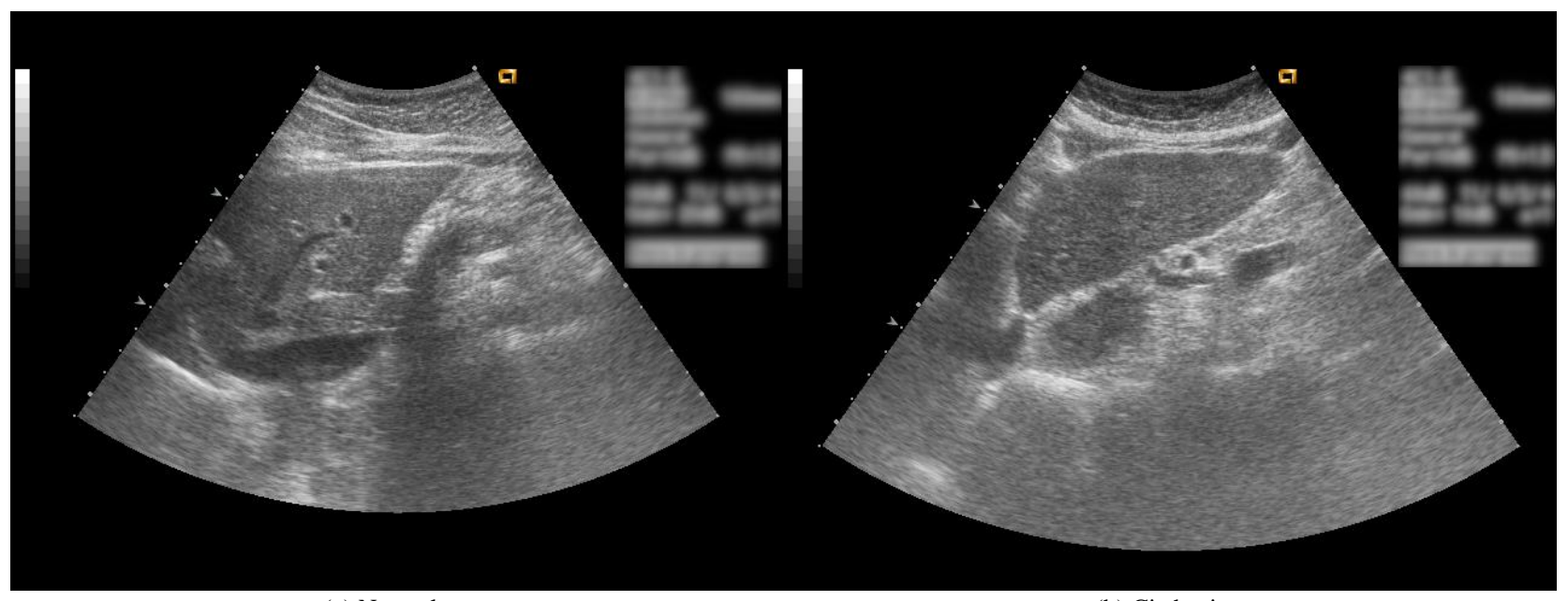

(a) Normal Fig. 1. B-mode ultrasound images.

(b) Cirrhosis

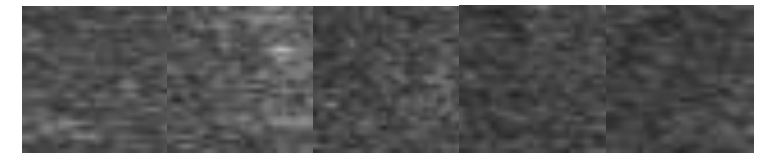

(a)Normal

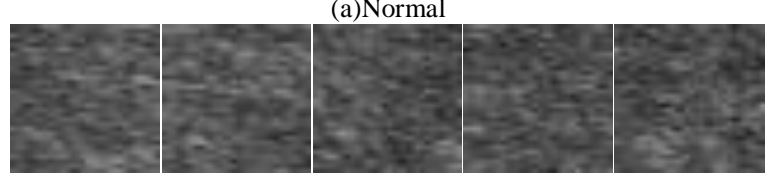

(b)Cirrhosis

Fig. 2. ROI images.

In this paper, in order to improve cirrhosis liver classification performance on ROI images from B mode ultrasound images, we propose to use a CNN. We further investigate an augmentation of the ROI images. The experimental results show the effectiveness of the CNNs. Furthermore, by using an augmentation technique, the classification performance of the CNNs is improved.

This paper is organized as follows: In the second section, we discuss the previous work. The third section describes our methodology. The fourth is the experiments. The experimental results show the effectiveness of the CNNs compared to the conventional method. The fifth is the conclusion.

\section{Previous WORK}

In order to classify the cirrhosis liver of B-mode ultrasound ROI images, the conventional approach focused on which features we should use. In a previous study [6], we compared the classification performance of Gabor features with that of HLAC features, because these features are preferable for texture images, like ultrasound images. For simplicity, we used a nearest neighbor classifier [13], [14]. And we further investigated how the HLAC feature could be improved by using some image processing techniques [15]. The adaptive thresholding technique [16] was most effective. Table I shows the average error rates of the conventional method. The figures show average error rate and the 95\% confidence interval. The HLAC feature* means the HLAC feature was improved with some image processing techniques. From the previous study, we found adaptive thresholding best for the classification. From the result, we could see the best performance of HLAC feature*, $44.1 \%$. However, even the best average error rate was still over $40 \%$, which is too low for clinical use. Therefore, we have to consider another approach such as the CNNs.

TABLE I: THE AVERAgE ERror RATES OF THE CONVENTIONAL METHOD, WITH 95\% CONFIDENCE INTERVALS

\begin{tabular}{|c|c|c|}
\hline Gabor feature & HLAC feature & HLAC feature* \\
\hline $48.3 \pm 0.6 \%$ & $47.6 \pm 0.5 \%$ & $\mathbf{4 4 . 1 \pm 0 . 5 \%}$ \\
\hline
\end{tabular}

\section{Methodology}

We describe the $\mathrm{CNN}$ and augmentation we used.

\section{A. CNN Architecture}

First, we show the CNN architecture. The performance of CNNs depends on its network structure, learning method, the parameters to be determined, and so on. In preliminary experiments, we have decided these. We used the CNN shown in Fig. 3. The input of the $\mathrm{CNN}$ is the ROI image of size $32 \times 32$. Firstly, we convolve the ROI image by using 32 filters with a $3 \times 3$ filter size. And by $2 \times 2$ maxpooling, we reduce the ROI image size to a half-sized image, $16 \times 16$. Secondly, we repeatedly convolve and do max-pooling in the same manner. Then, we get $328 \times 8$ sized image. Thirdly, we flatten this image into $2,048(=32 \times 8 \times 8)$-dimensional data Finally, we make a fully connected artificial neural network. 


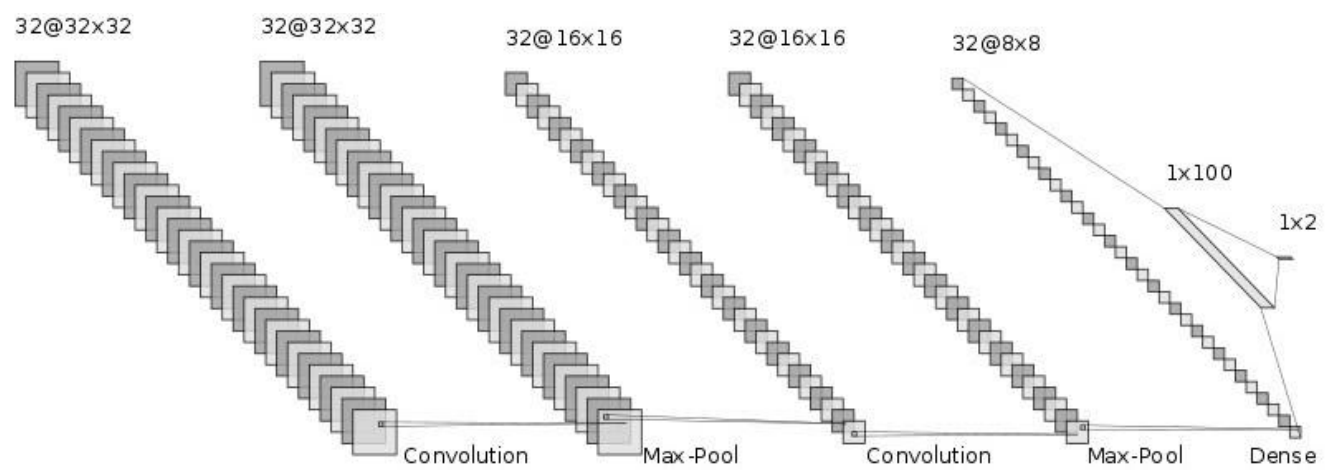

Fig. 3. A network structure of $\mathrm{CNN}$ we used.

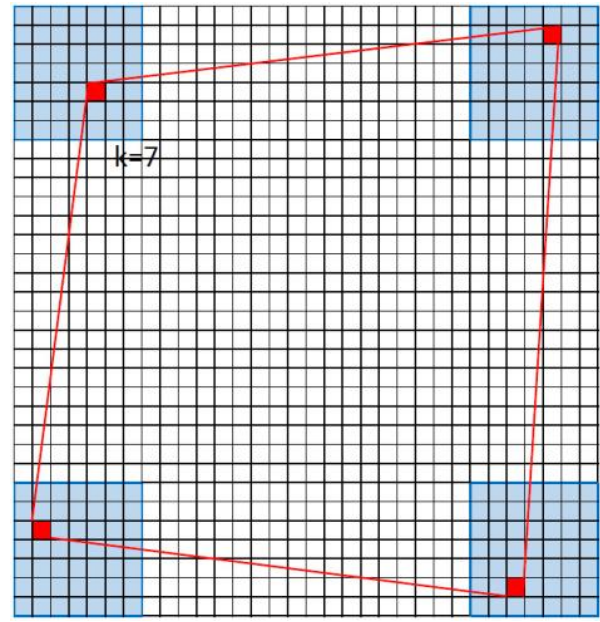

(a) Before transformation

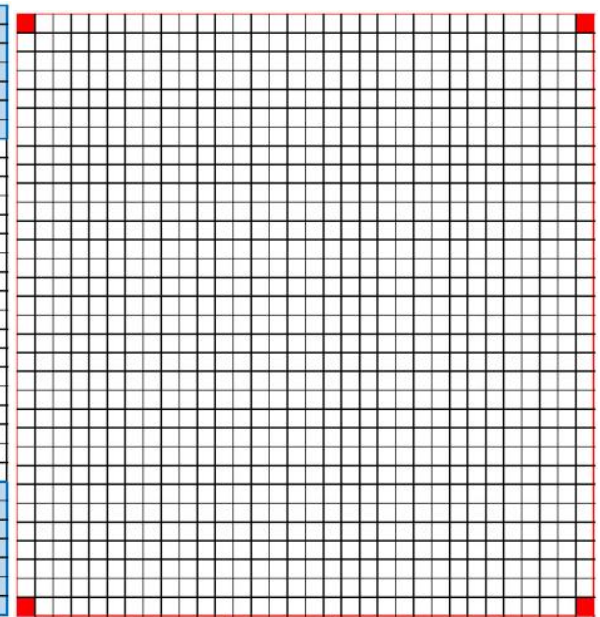

(b) After transformation

Fig. 4. An illustration of a perspective transformation. The quadrilateral bounded by the left 4 red points is mapping by a homography of the right image.

The network has one hidden layer. The number of the neurons also depends on the classification performance. For simplicity, we used 100 . Then we used dropout. The rate of dropout is 0.5 . The number of the outputs of the CNN is 2 . This corresponds to a 2-class problem. Therefore, the structure of the fully connected artificial neural network is 2,048-100-2. All the activation functions are ReLU except for the output. In the output, we used softmax. The learning optimizer is adam. The epochs and batch size are 100 and 400, respectively.

\section{B. Augmentation Techniques}

Here, we show an augmentation technique we used. In general, CNNs need a lot of samples to train properly. On the other hand, the number of the ROI images is limited in our study. Therefore, we generate additional samples by using the augmentation technique. In this study, we have tried using a perspective transformation.

We describe a perspective transformation, which has some distortion. We can see a lot of images which differ their appearance from an image by the perspective transformation. The equation of the perspective transformation is define by the following.

$$
\begin{aligned}
x_{i}^{\prime} & =\frac{a_{1} x_{i}+a_{2} y_{i}+a_{3}}{a_{7} x_{\mathrm{i}}+a_{8} y_{i}+1} \\
\mathrm{y}_{\mathrm{i}}^{\prime} & =\frac{a_{4} \mathrm{x}_{\mathrm{i}}+a_{5} \mathrm{y}_{\mathrm{i}}+a_{6}}{a_{7} x_{i}+a_{8} y_{i}+1}
\end{aligned}
$$

The notation $(x, y)$ denotes a point of an original image. On the other hand, $\left(x^{\prime}, y^{\prime}\right)$ is a point of an image newly generated by the perspective transformation given by the parameter values of $a_{1}-a_{8}$. The newly generated image is usually a little smaller than an original image. The appearance seems a little larger. Fig. 4 shows an illustration of a perspective transform. Fig. 4(a) shows an illustration from before transformation. In the perspective transformation, we need at least 4 corresponding points between the before and after transformed images. The parameter values $\mathrm{a}_{1}-\mathrm{a}_{8}$ could be computed by this corresponding relationship. These values $a_{1}-a_{8}$ were calculated by solving a simultaneous linear equation. In the experiments, we used 4 randomly generated points, such as in the light blue corners in Fig. 4(a). In each of these areas, we choose a random point. In this case, we can see a selection box of size $k=7$. In the experiments, we used $k$ $=3,5,7$, and 9. Every trial, we get an artificially generated new image. We can get an arbitrary number of images artificially generated. Given 400 training images, we could get 400 $\times x$ training images. In the experiments, we used $x=8$, 24,48 , and 128 . Therefore, we used $3,200,9,600,19,200$, and 51,200 training images.

\section{EXPERIMENTS}

We describe the experiments we conducted.

\section{A. Evaluation of the Generalization Performance of the CNN}

In the experiment, we used 500 available ROI images: 200 normal images and 300 cirrhosis images. This is a 2-class problem as mentioned previously. The gray level is 8 bits. The effectiveness of the CNNs is examined in terms of the error rate. The error rate is defined as a ratio of the number of 
test images misclassified to the number of all test images.

$$
E=\frac{\text { No.test images misclassified }}{\text { No.all test images }} \times 100(\%)
$$

For error rate estimation, the holdout method has been successfully used, because it maintains the statistical independence between the training and test images [13], [17]. To evaluate the classification performance, i.e. generalization performance of the $\mathrm{CNN}$, the average error rate was obtained by the holdout method. Fig. 5 shows the flow of the error rate estimation. First, we randomly divided the 500 available ROI images into 400 training ROI images and 100 test ROI images. The 400 training ROI images consist of 160 normal and 240 cirrhosis ROI images. The 100 test ROI images consist of 40 normal and 60 cirrhosis ROI images. Second, we augmented the training images as described above. Third, we train the $\mathrm{CNN}$ using the training images and compute the error rate using the test images. Finally, by 10 repetitions, the average error rate and $95 \%$ confidence interval were obtained. Fig. 5 illustrates the method used.

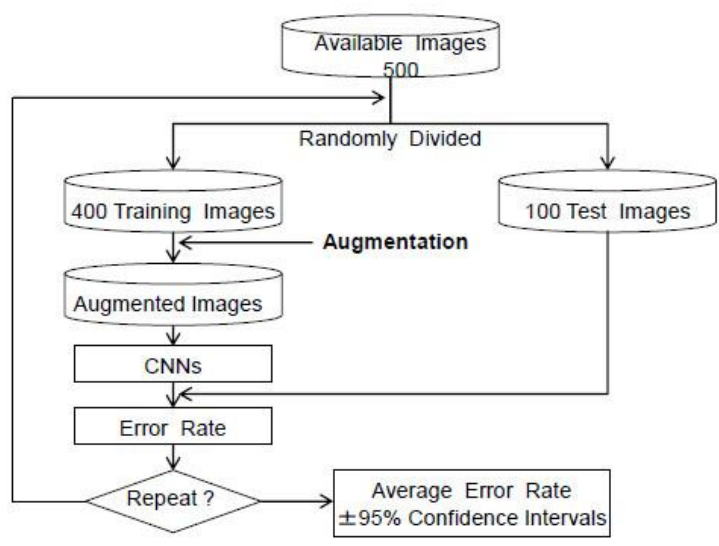

Fig. 5. A flow of the error rate estimation.

\section{B. Experiment 1}

The purpose of experiment 1 is to investigate the classification performance of the $\mathrm{CNN}$ by using gray and binary ROI images in terms of the error rate. From the previous study, we found that binary images were effective for classification. Therefore, we have conducted this experiment. The binarization method was the adaptive thresholding method [16]. Table II shows the average error rate of the CNN by using the gray and binary ROI images. From Table II, we see the gray ROI images are superior to the binary ones. Therefore, all remaining experiments were conducted using the gray ROI images.

TABLE II: THE AVERAGE ERROR RATES OF THE CNN WHEN USING GRAY

\begin{tabular}{|c|c|}
\hline \multicolumn{2}{|c|}{ AND BINARY ROI IMAGES } \\
\hline Gray & Binary \\
\hline $\mathbf{3 4 . 4} \pm \mathbf{3 . 5} \%$ & $42.4 \pm 4.1 \%$ \\
\hline
\end{tabular}

\section{Experiment 2}

The purpose of experiment 2 is to investigate effects of the augmentation by a perspective transformation. The image perspectively transformed has some distortion. But we can generate many samples by this technique. We can control the number of images artificially generated. In the experiments, the numbers of images augmented by the perspective transformation including original images are 3,200 $(=400 \times 8)$, $9,600(=400 \times 24), 19,200(=400 \times 48)$, and 51,200 $(=400 \times 128)$, respectively. From the experimental results, we chose $k=7$ which determines the areas with 4 points randomly generated. Refer to the light blue areas in the Fig. 4. Table III shows the result of the average error rates of the $\mathrm{CNN}$ for each number of augmentations by the perspective transformation. From Table III, we see the average error rate, $31.9 \%$, is the lowest when the number of the images augmented is 9,600. The average error rate of the $\mathrm{CNN}$ with this augmentation outperforms that of the CNN without augmentation.

TABLE III: THE AVERAGE ERROR RATES OF THE CNN FOR EACH OF THE

\begin{tabular}{|c|c|c|c|}
\hline \multicolumn{4}{|c}{ NUMBERS OF THE AUGMENTATION } \\
\hline 3,200 & 9,600 & 19,200 & 51,200 \\
\hline $35.1 \pm 2.1 \%$ & $\mathbf{3 1 . 9} \pm \mathbf{2 . 5 \%}$ & $34.9 \pm 3.2 \%$ & $34.2 \pm 3.4 \%$ \\
\hline
\end{tabular}

\section{Experiment 3}

The purpose of experiment 3 is to investigate effects of the parameter value $\mathrm{k}$ of the perspective transformation in terms of the average error rate. We expect the parameter value $\mathrm{k}$ influences the generalization performance of the CNN. In the experiment, the number of augmented images are 19,200. This situation is the same of experiment 2 . We conducted the experiment using $k=3,5,7$, and 9. Table IV shows the average error rate of the $\mathrm{CNN}$ for each of values of $\mathrm{k}$. From Table IV, we see the average error rate using $k=7$ was the lowest in our experiment. There seems an optimal value of $k$ in terms of the average error rate. The average error rate goes worse if the value of $\mathrm{k}$ is too larger or smaller. If we could select an optimal value of $\mathrm{k}$ of the perspective transformation, we should try examining some candidates of $\mathrm{k}$. In the perspective transformation, we should take care of a selection of a value of $\mathrm{k}$.

TABLE IV: THE AVERAGE ERROR RATES OF THE CNN FOR Each OF Values

\begin{tabular}{|c|c|c|c|}
\hline \multicolumn{4}{|c|}{ OF $K$} \\
\hline $33.6 \pm 3.3 \%$ & $k=5$ & $k=7$ & $k=9$ \\
\hline
\end{tabular}

\section{E. Experiment 4}

The purpose of experiment 4 is to investigate a difference of the CNN without and with augmentation. We used augmentation by the perspective transformation of $k=7$. To investigate the difference, we compared both the confusion matrices. The confusion matrix is a detail of 10 classified or misclassified average numbers out of all the test images. The numbers of training and test images were the same as the experiment described here. As mentioned above, the numbers of normal and cirrhosis test images were 40 and 60 , respectively. Table $\mathrm{V}$ shows the confusion matrices of 10 average numbers of test images. Table V(a) and Table V(b) are the CNNs without and with the augmented images. In these tables, the left side classes show the test classes. The top side classes show the classes to be classified or misclassified. The diagonal numbers mean the 10 correct classified average number of test images. On the other hand, the nondiagonal numbers mean the 10 misclassified average number of test images. Ideally the first and second diagonal numbers should be 40 and 60, respectively. The nondiagonal number should be 0 . Comparing (a) to (b), we see all the diagonal numbers 
decrease by the use of augmented images. Furthermore, we see the improvement that the normal images goes wrong to the cirrhosis class. On the other hand, we also see the defect that the cirrhosis images goes wrong to the normal class. Therefore, we should pay attention to this situation in using CNN with augmentation.

TABLE V: THE CONFusion Matrices OF 10 AVERAGE Numbers OF TEST IMAGES

(A) CNN WiTHouT AugMENTED IMAGES

\begin{tabular}{|c|r|r|}
\hline \multirow{2}{*}{$\begin{array}{c}\text { Test } \\
\text { classes }\end{array}$} & \multicolumn{2}{|c|}{ Classes to be classified or misclassified } \\
\cline { 2 - 3 } & Normal & \multicolumn{1}{c|}{ Cirrhosis } \\
\hline Normal & 10.6 & 27.3 \\
\hline Cirrhosis & 7.1 & 55.0 \\
\hline
\end{tabular}

(B) CNN With AugMented IMAges By THE PERSPECTIVE TRANSFORMATION USING $K=7$

\begin{tabular}{|c|c|c|}
\hline \multirow{2}{*}{$\begin{array}{c}\text { Test } \\
\text { classes }\end{array}$} & \multicolumn{2}{|c|}{ Classes to be classified or misclassified } \\
\hline & Normal & Cirrhosis \\
\hline Normal & 21.7 & 16.9 \\
\hline Cirrhosis & 15.0 & 46.4 \\
\hline
\end{tabular}

\section{F. Summary of Our Experimental Results}

We summarize our proposed method as we mention each of our experimental results.

1) We should use the gray ROI image not binary one using the $\mathrm{CNN}$.

2) We should find the optimal number of the augmented images in terms of the average error rate.

3) The value of $\mathrm{k}$ in the perspective transformation need to be selected carefully in terms of the average error rate.

4) We should take care of the detail which type of the error occurred.

From all the experimental results, we see the best average error rate of CNNs is $31.9 \%$. This significantly outperforms the conventional method by HLAC features with the average error rate, $44.1 \%$. It is significantly better in terms of t-test, $p$ $\leq 0.01$.

\section{CONCLUSION}

In order to accurately classify the cirrhosis liver ultrasound ROI images, we have explored the use of CNNs. Furthermore, by an augmentation technique, the classification performance of the CNNs has been increased. The experimental results show that the classification performance of the $\mathrm{CNN}$ outperforms that of the conventional method, when using data augmentation by a perspective transformation technique. As we expected, the CNN has produced a dramatic improvement even with a difficult pattern recognition problem such ultrasound ROI image classification.

When applying CNNs to this problem, we could consider other network structures, parameter settings, other learning methods, and so on. In the future, we would get more real data and explore the whole ultrasound images such as Fig. 1 by using CNNs. Furthermore, we should try other augmentation techniques, such as by adding small Gaussian noise to images. There is a generative adversarial network (GAN) [18] which automatically generates fake samples. By using this technique, the fake images seem very similar to the real. Moreover, the deep convolutional generative adversarial networks (DCGANs) [19] was published recently. This technique is possibly preferable. In the future, we will investigate applying DCGANs to the cirrhosis liver classification problem.

\section{CONFLICT OF INTEREST}

The authors declare no conflict of interest.

\section{AUTHOR CONTRIBUTIONS}

Yoshihiro Mitani, Robert B. Fisher, Yusuke Fujita, Yoshihiko Hamamoto, and Isao Sakaida concuted the research; Yoshihiro Mitani, Robert B. Fisher, and Yusuke Fujita analyzed the data; Yoshihiro Mitani and Robert B. Fisher wrote the paper; Yoshihiko Hamamoto and Isao Sakaida created the research framework; All authors had approved the final version.

\section{REFERENCES}

[1] K. Doi, "Computer-aided diagnosis in medical imaging: Historical review, current status and future potential," Computerized Medical Imaging and Graphics, vol. 31, pp. 198-211, 2007.

[2] A. Huber, L. Ebner, J. T. Heverhagen, and A. Christe, "State-of-the-art imaging of liver fibrosis and cirrhosis: A comprehensive review of current applications and future perspectives," European Journal of Radiology Open, vol. 2, pp. 90-100, 2015.

[3] Y. Fujita, Y. Hamamoto, M. Segawa, S. Terai, and I. Sakaida, "An improved method for cirrhosis detection using liver's ultrasound images," in Proc. 20th International Conference on Pattern Recognition, 2010, pp. 2294-2297.

[4] Y. Fujita, T. Goto, Y. Hamamoto, Y. Mitani, M. Segawa, S. Terai, and I. Sakaida, "A liver cirrhosis detection method using probabilistic ROI combination," in Proc. 11th International Conference on Quality Control by Artificial Vision, 2013, pp. 81-85.

[5] Y. Fujita, Y. Mitani, Y. Hamamoto, M. Segawa, S. Terai, and I. Sakaida, "Training sample selection based on self-training for liver cirrhosis classification using ultrasound images," in Proc. 13th International Conference on Quality Control by Artificial Vision, 2017

[6] K. Sasaki, Y. Mitani, Y. Fujita, Y. Hamamoto, and I. Sakaida, "A liver cirrhosis classification on b-mode ultrasound images by the use of higher order local autocorrelation features," in Proc. 8th International Conference on Graphic and Image Processing, 102250U, 2017.

[7] D. E. Rumelhart, G. E. Hinton, and R. J. Williams, "Learning representations by back propagating errors," Nature, vol. 323, no. 9, pp. 533-536, 1986.

[8] G. E. Hinton, S. Osindero, and Y. W. Teh, "A fast learning algorithm for deep belief nets," Neural Computation, vol. 18, no. 7, pp. 1527-1554, 2006.

[9] Y. LeCun, Y. Bengio, and G. Hinton, "Deep learning, "Nature, vol. 521, no. 28, pp. 436-444, 2015.

[10] G. Litjens, T. Kooi, B. E. Bejnordi, A. A. A. Setio, F. Ciompi, M. Ghafoorian, J. A. W. M van der Laak, B. van Ginneken, and C. I. Sanchez, "A survey on deep learning in medical image analysis," Medical Image Analysis, vol. 42, pp. 60-88, 2017.

[11] S. J. Raudys and A. K. Jain, "Small sample size effects in statistical pattern recognition: Recommendations for practitioners and open problem," in Proc. 10th International Conference on Pattern Recognition, 1990, pp. 417-423.

[12] S. J. Raudys and A. K. Jain, "Small sample size problems in designing artificial neural networks," Machine Intelligence and Pattern Recognition, vol. 11, pp. 33-50, 1991.

[13] R. O. Duda, P. E. Hart, and D. G. Stork, Pattern Classification, Second Edition, Wiley Interscience, 2001.

[14] T. M. Cover and P. E. Hart, "Nearest neighbor pattern classification," IEEE Trans., IT-13, pp. 21-27, 1967.

[15] J. C. Russ, The Image Processing Handbook, Third Edition, CRC Press, 1990.

[16] R. Fisher, S. Perkins, A. Walker, and E. Wolfart. (2003). Adaptive thresholding. [Online]. Available: https://homepages

[17] K. Fukunaga, Introduction to Statistical Pattern Recognition, Second Edition, Academic Press, 1990.

[18] I. J. Goodfellow, J. Pouget-Abadie, M. Mirza, B. Xu, D. Warde-Farley, S. Ozair, A. Courville, and Y. Bengio, "Generative adversarial nets," in Proc. Advances in Neural Information Processing Systems, 2014, vol. 27. 
[19] A. Radford, L. Metz, and S. Chintala, "Unsupervised representation learning with deep convolutional generative adversarial networks," in Proc. 2016 International Conference on Learning Representations, 2016.

Copyright $\odot 2020$ by the authors. This is an open access article distributed under the Creative Commons Attribution License which permits unrestricted use, distribution, and reproduction in any medium, provided the original work is properly cited (CC BY 4.0).

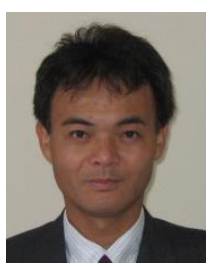

Yoshihiro Mitani is currently a professor at National Institute of Technology, Ube College, Japan. His research interests include pattern recognition and image processing techniques. He is a member of IEEE.

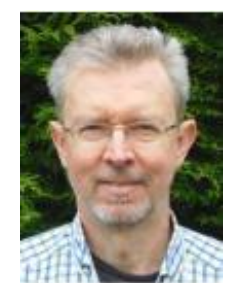

Robert B. Fisher is currently a professor at the University of Edinburgh, UK. His research interests include computer vision. The main topics of his recent research are vision for human-robot interaction, and outdoor robotics. He is the leader of the TrimBot2020 gardening robot project.

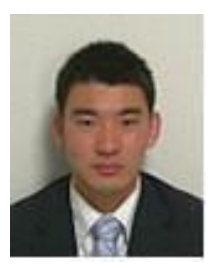

Yusuke Fujita is currently an associate professor at Yamaguchi University, Japan. His research interests include machine learning, image processing, pattern recognition and computer vision.

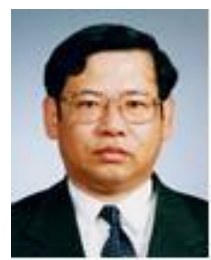

Yoshihiko Hamamoto is currently a professor at Yamaguchi University, Japan. His research interests include pattern recognition. His research area is informatics, intelligent informatics. He is a member of IEEE.

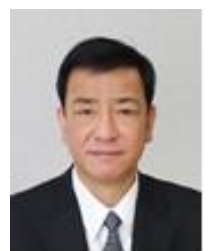

Isao Sakaida is currently a medical doctor and a professor at Yamaguchi University, Japan. His research interests include liver disease. His research area is clinical internal medicine, gastroenterology. 